\title{
Testbed for on-orbit servicing and formation flying dynamics emulation
}

\author{
Markus Schlotterer* and Stephan Theil ${ }^{\dagger}$ \\ DLR, Institute of Space Systems, Bremen, D28359, Germany
}

\begin{abstract}
The testbed for on-orbit servicing and formation flying dynamics emulation is a facility to emulate the force and momentum-free dynamics of multi-spacecraft missions on ground. The facility consists of a large granite table and several air cushion vehicles that float on this table. The granite table has a size of $4 \mathrm{~m} \times 2.5 \mathrm{~m}$ and a thickness of $0.6 \mathrm{~m}$ with a very high evenness.

The air-cushion vehicles consist of two parts: a lower translation platform and an upper attitude platform. Both platforms are connected by a spherical air bearing. The translation platform carries the flat air bearings which enable the vehicle to float on the table, high pressure air tanks to support the flat and the spherical air bearings and a vertical linear actuator. With this actuator it is possible to adjust the altitude of the spherical air bearing and thereby the altitude of the attitude platform.

All components needed for a control loop to actuate the air cushion vehicles are mounted on the attitude platform. These are 12 proportional cold gas thrusters with high pressure air tanks, 3 reaction wheels, an onboard computer with real-time operating system and Wireless LAN for communications and software upload, an inertial measurement unit (IMU) and a sensor for an infrared tracking system. In addition the attitude platform carries a power system with batteries to support all components with electrical energy and a balancing system to move the center of mass in the center of the spherical air bearing.

Due to the linear air bearing the system can emulate 2 translational degrees of freedom. The third translational degree of freedom (parallel to gravity vector) can be actuated by the vertical linear actuator. As the attitude platform can tilt and rotate, 3 rotational degrees of freedom are emulated. So there is a total of $5+1$ degrees of freedom that can be emulated by the testbed.

One purpose of this testbed is the testing of control algorithms for multi-spacecraft missions. It can be used for precise formation control as well as path-planning and formation acquisition. In addition to the usage of real hardware as in Hardware-in-the-Loop simulations, the force and momentum-free dynamics are not simulated but are real (emulation). This is also a foundation for the second task of the testbed: the emulation of contact dynamics for Rendezvous and Docking missions. Models of docking adapters can be mounted on the attitude platform of the air-cushion vehicles and docking maneuvers with real contact dynamics can be tested on ground. A further purpose of the testbed is the qualification of dedicated relative navigation sensors and inter-spacecraft communication systems. These components can also be mounted on the attitude platform and tested in an agile environment.

This paper describes the design and configuration of the testbed for on-orbit servicing and formation flying dynamics emulation. Results showing the quality of the force and momentum-free environment are presented. First control, navigation and thruster actuation algorithms have been developed and their design and the result of their usage within the testbed are also shown. Finally possible scenarios for the usage of this testbed are presented and an outlook on further developments and improvements is given.
\end{abstract}

\footnotetext{
*GNC Engineer, GNC Systems, DLR Institute of Space Systems, Robert-Hooke-Str. 7, 28359 Bremen, Germany, AIAA Member.

${ }^{\dagger}$ Head of GNC Systems Department, DLR Institute of Space Systems, Robert-Hooke-Str. 7, 28359 Bremen, Germany, AIAA Member.
} 


\section{Introduction}

The size of today's satellites is limited because of the shipping volume of the aerospace transportation system used. To improve the earth observation or the resolution of telescopes however larger and larger structures are necessary. To overcome this restriction, more and more satellite formations are to adopt in years to come. These are made up of two or more single satellites.

Two examples of this kind of application are the ESA mission study DARWIN ${ }^{1}$ and the planned ESA X-ray observatory XEUS. ${ }^{2}$ DARWIN was designed as a constellation of several satellites with one central satellite to search for Earth-like planets around other stars (see figure 1). For that DARWIN uses a technique called "nulling interferometry". By adjusting the phase difference of the light reaching different telescopes and controlling it to zero the central star does not show up in the combined image. As the direction to the planet is not the same as to the central star the phase difference of the light from the planet to different telescopes is not zero and such the planet can be seen in the combination of the images from different telescopes. To fulfill this task the constellation has to stay in formation with millimeter precision.

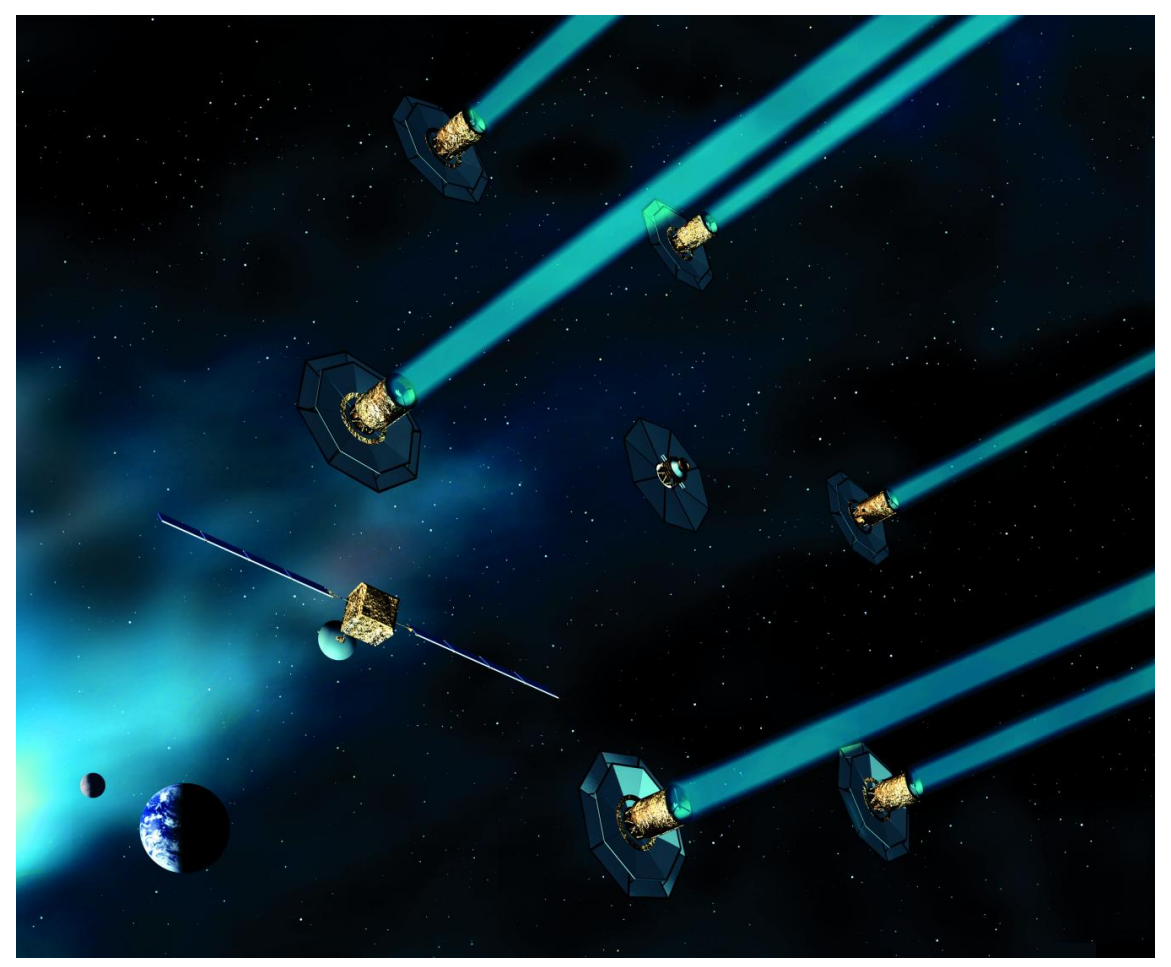

Figure 1. Satellite constellation DARWIN, Image: ESA

XEUS will be a free-flying X-ray telescope consisting of 2 satellites with the mirror on one spacecraft and the detector on the other. The 2 satellites will stay in formation $50 \mathrm{~m}$ apart and their relative position will have to be controlled with a precision of $1 \mathrm{~mm}$.

In addition there are upcoming missions and feasibility studies that use Rendezvous and Docking for several tasks like On-Orbit Servicing (OOS) or space debris removal. One example in the field of OOS is the first German servicing mission (Deutsche Orbitale Servicing Mission, DEOS). ${ }^{3}$ DEOS is a demonstrator mission for several tasks in the field of Rendezvous and Docking. The main goals of this mission are the rendezvous to and berthing of a non-cooperative and tumbling spacecraft by means of a manipulator system accommodated on a servicing satellite, the docking of the spacecraft via a dedicated docking device and servicing tasks in the coupled configuration as well as the controlled de-orbiting / re-entry of the spacecraft's coupled configuration in the Earth's atmosphere at the end of the mission.

All these ideas, studies and planned missions have in common that they use two or more satellites that fly in a formation or perform a Rendezvous \& Docking maneuver. To study the relative and maybe contact dynamics of such spacecrafts and to test formation control algorithms and relative navigation sensors the 


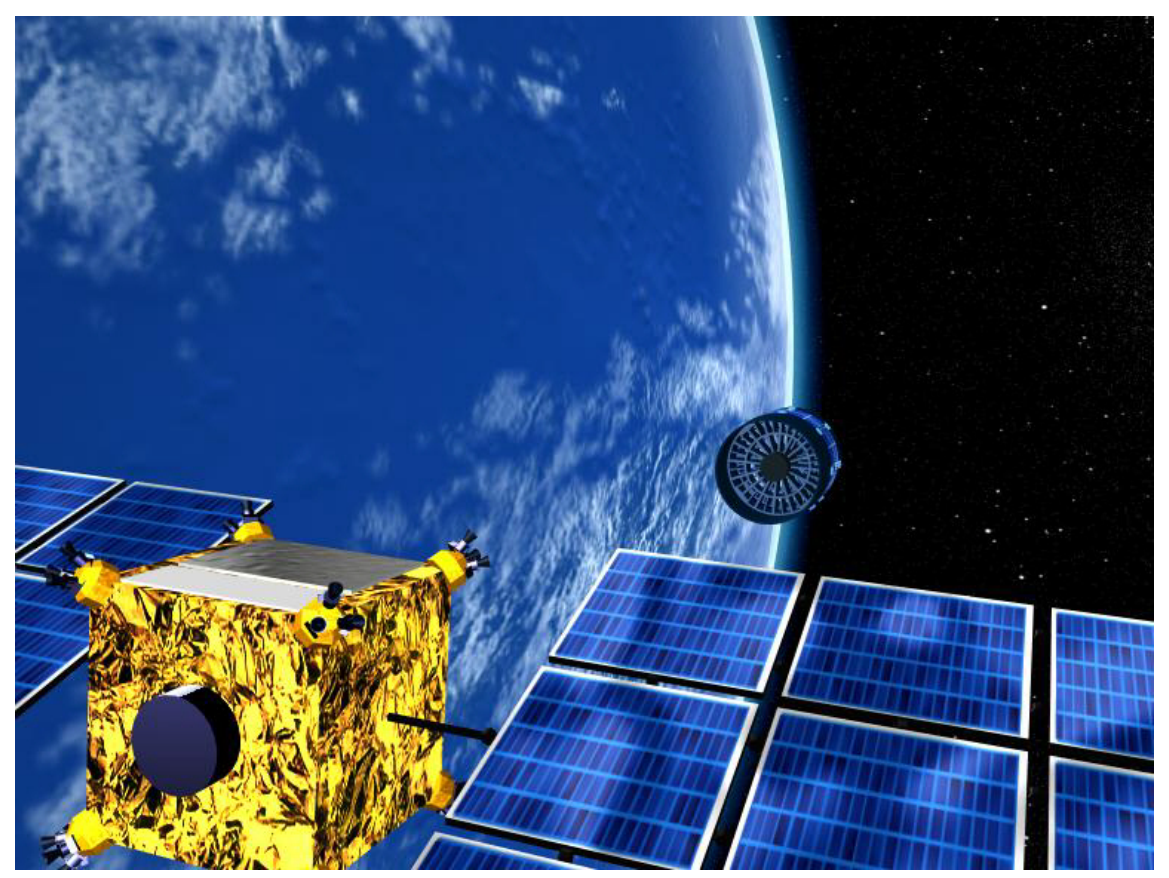

Figure 2. X-ray observatory XEUS, Image: ESA

Institute of Space Systems of the German Aerospace Center (DLR) develops a facility called the Formation Flying Testbed (FFT).

The FFT consists of a large granite table with a high evenness and several air cushion vehicles floating on the table. These vehicles emulate the satellites of the formation. Due to the air cushion the vehicles perform a nearly force-free motion in two degrees of freedom. The upper part of the vehicles, the so called Attitude Platform, is supported by a spherical air bearing. Thereby the Attitude Platform can perform a momentum-free motion in 3 degrees of freedom.

The first purpose of the testbed is the test of control algorithm for formation flying and Rendezvous and Docking missions. Contrary to Hardware-in-the-Loop test platforms, the dynamics of the spacecrafts are real. A second purpose is the possibility to emulate contact dynamics while docking or berthing with another satellite. Models of docking mechanisms or manipulator arms can be mounted on the upper platform and the dynamical behavior of the satellites during the docking or berthing maneuver can be tested. Last but not least it is also possible to mount relative navigation sensors on the vehicles and test their behavior in a dynamic environment.

\section{The Formation Flying Testbed}

The Formation Flying Testbed consists of a large granite table with several air cushion vehicles floating on the table (figure 3). The size of the table is $4 \times 2.5 \mathrm{~m}$. The surface has been manufactured with an accuracy of $3 \mu \mathrm{m}$. Concerning the orientation to the gravitational field the granite table has been leveled with an accuracy of less than $20 \mu \mathrm{m}$ from one edge to the other. The high evenness and the leveling are necessary to guarantee that the disturbing force due to the gravitational field is very small. The thickness of the granite is $60 \mathrm{~cm}$, a value necessary to keep the high evenness in presence of the load due to the air cushion vehicles and the granite table's own weight.

The air cushion vehicles consist of two parts (see figure 4). The lower part is called the Transport Platform. At the bottom of the platform the air cushion pads support the platform in a force-free way on the granite table. The platform also consists of the air tanks and the pressure regulators to support the pads and the spherical air bearing with pressurized air. In the middle of the platform an actuated linear stage is mounted. With this stage it is possible to adjust the height of the vehicles. On the top of the stage the spherical air bearing acts as joint between the Transport Platform and the upper so-called 


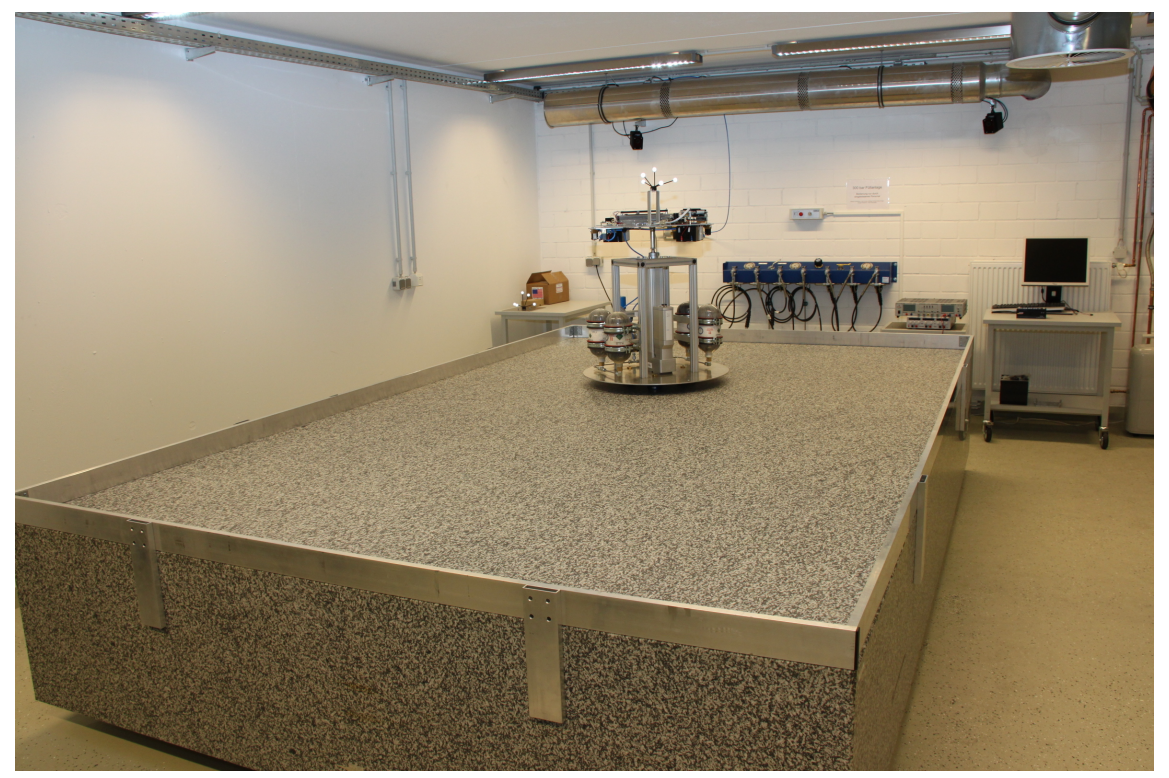

Figure 3. The Formation Flying Testbed

Attitude Platform. There is no other connection between the Attitude and the Transport Platform than this spherical air bearing. So the Attitude Platform can move in a momentum-free motion around this joint.

The Attitude Platform carries tanks and pressure regulators, too. These are necessary to support the thrusters with pressurized air. In addition all components for a complete control loop are mounted on this platform. As actuators there are 12 thrusters to generate forces and torques and 3 reaction wheels for fast attitude control. To measure position and attitude but also acceleration and attitude rate an infrared-based tracking system and an inertial measurement unit (IMU) are used (see section II.B). To process the measured data and to drive the actuators there is also an onboard computer with WLAN adapter for commanding, software upload and data download. To support all components with electrical energy a battery system with power distribution unit (PDU) is also part of the upper platform.

\section{A. Air system}

On the Transport Platform as well as on the Attitude Platform there is an air supply system to support the air cushion pads, the spherical air bearing and the thrusters with pressurized air. The air supply system consists of 300 bar air tanks and several pressure regulators. The air tanks are made of glass-fibre reinforced plastic (GRP) with an aluminum insert. On the Transport Platform there are $4 \times 1.5 \mathrm{l}$ tanks, on the Attitude Platform $4 \times 1.11$ tanks. A coarse pressure regulator is part of the Transport Platform as well as of the Attitude Platform. This regulator regulates the high pressure air coming from the tanks (between 20 and 300 bar) down to about 12 bar. For the thrusters, the air cushion pads and the spherical air bearing dedicated fine pressure regulators are necessary for regulating the pressure down to 6-8 bar depending on the component that is to be supported.

\section{B. Sensors}

Two sensors are used on the vehicles of the Formation Flying Testbed: an inertial measurement unit (IMU) to measure accelerations and attitude rates and an infrared based tracking system to measure position and attitude of the vehicles.

\section{Inertial measurement unit}

The inertial measurement unit (IMU) consists of 3 accelerometers to measure acceleration and 3 gyroscopes to measure attitude rate. The IMU used for the vehicles of the Formation Flying Testbed is a $i V R U-F C$ from 


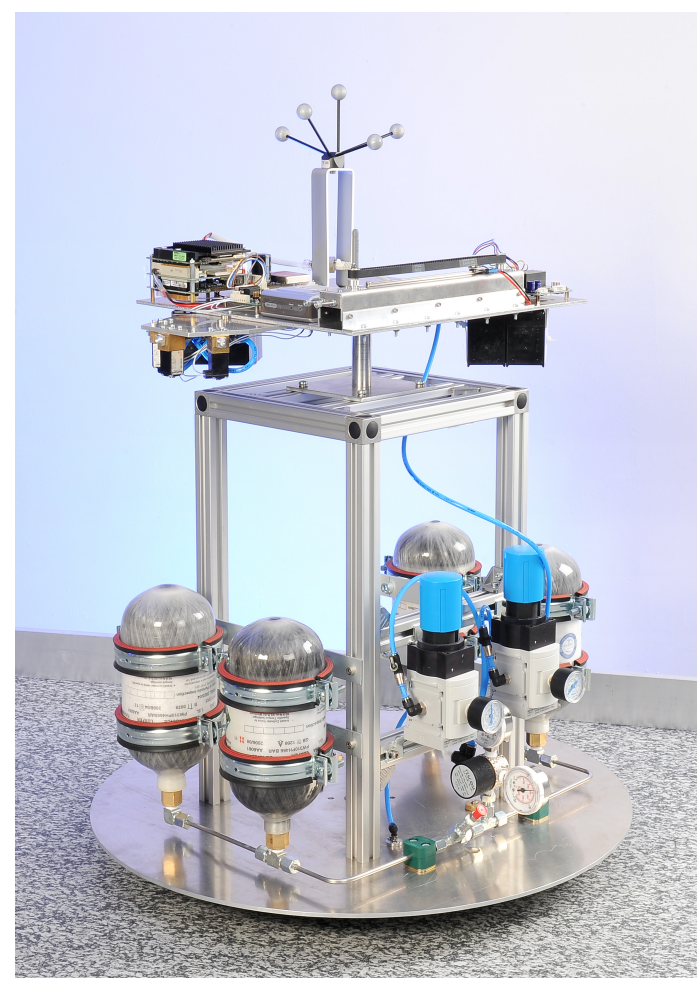

(a) Current status

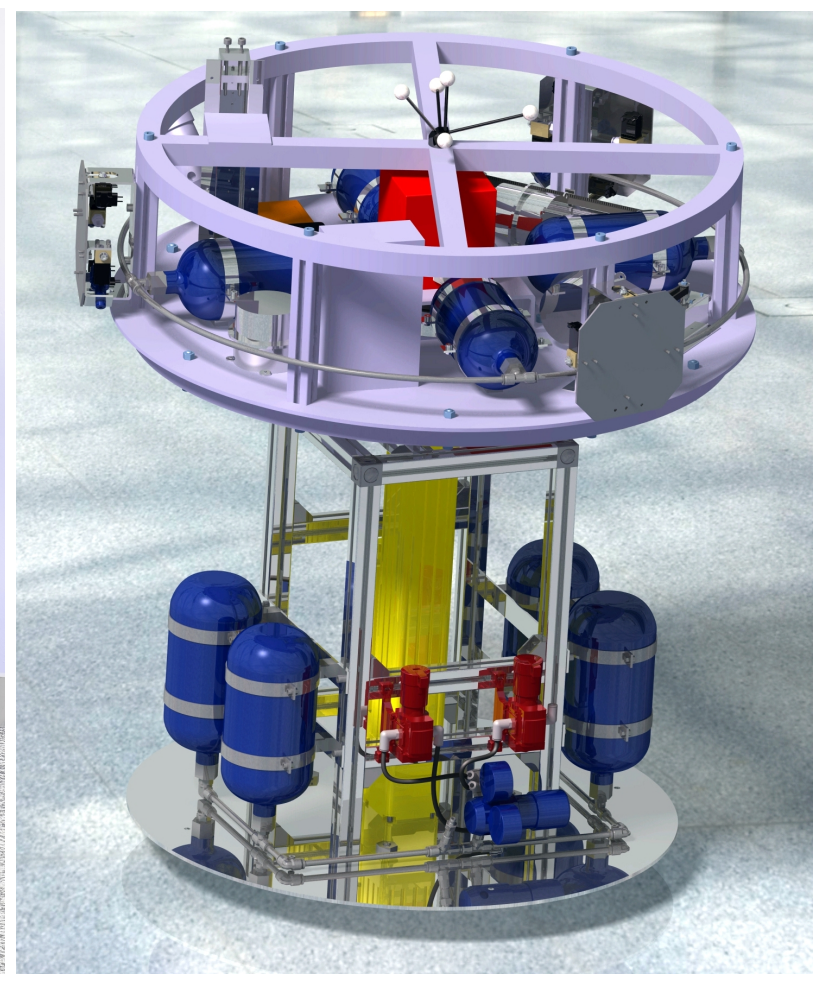

(b) Vehicle with Attitude Platform

Figure 4. The vehicles of the Formation Flying Testbed

the German manufacturer $i M A R$. It consists of three orthogonally mounted rugged fiber optic gyroscopes, three ultra ruggedized MEMS accelerometers and an integrated microprocessor. The technical specification of the IMU can be found in table 1.

Table 1. IMU technical specification

\begin{tabular}{|l|l|l|}
\hline & Gyroscopes & Accelerometer \\
\hline Sensor Range & $\pm 200 \% \mathrm{~s}$ & $\pm 2 \mathrm{~g}$ \\
Bias (const. temp., short time stability) & $<0.003^{\circ} / \mathrm{s}$ & $<0.5 \mathrm{mg}$ \\
Bias (long-term bias) & $<0.01 \%$ & $<2.5 \mathrm{mg}$ \\
Scale error & $<0.2 \%$ & $<0.3 \%$ \\
Noise (0-200 Hz) & $<0.1 \% \sqrt{\mathrm{Hz}}$ & $<15 \mu \mathrm{g} / \sqrt{\mathrm{Hz}}$ \\
Bandwidth & $0 \ldots 200 \mathrm{~Hz}$ & $0 \ldots 200 \mathrm{~Hz}$ \\
\hline
\end{tabular}

\section{DTrack infrared tracking system}

The infrared based realtime tracking system DTrack consists of 3 components:

- The tracking PC

- At least two tracking cameras and

- Up to 20 targets.

The targets are mounted on the object to be tracked and consist of several reflective balls, so-called markers. The tracking cameras emit infrared flashes with a frequency of $60 \mathrm{~Hz}$. The infrared light is reflected by the markers and detected by the cameras. The images from the different cameras are sent to the tracking PC 
which computes $6 \mathrm{DoF}$ target positions and orientations. The navigation solution is broadcasted on the local network and can be used by every computer that is connected via cable or wireless network.

For the Formation Flying Testbed a DTrack system with 4 cameras is used. After the mounting of the cameras the system can be easily calibrated using a calibration target. It is also easy to extend the system with more cameras.

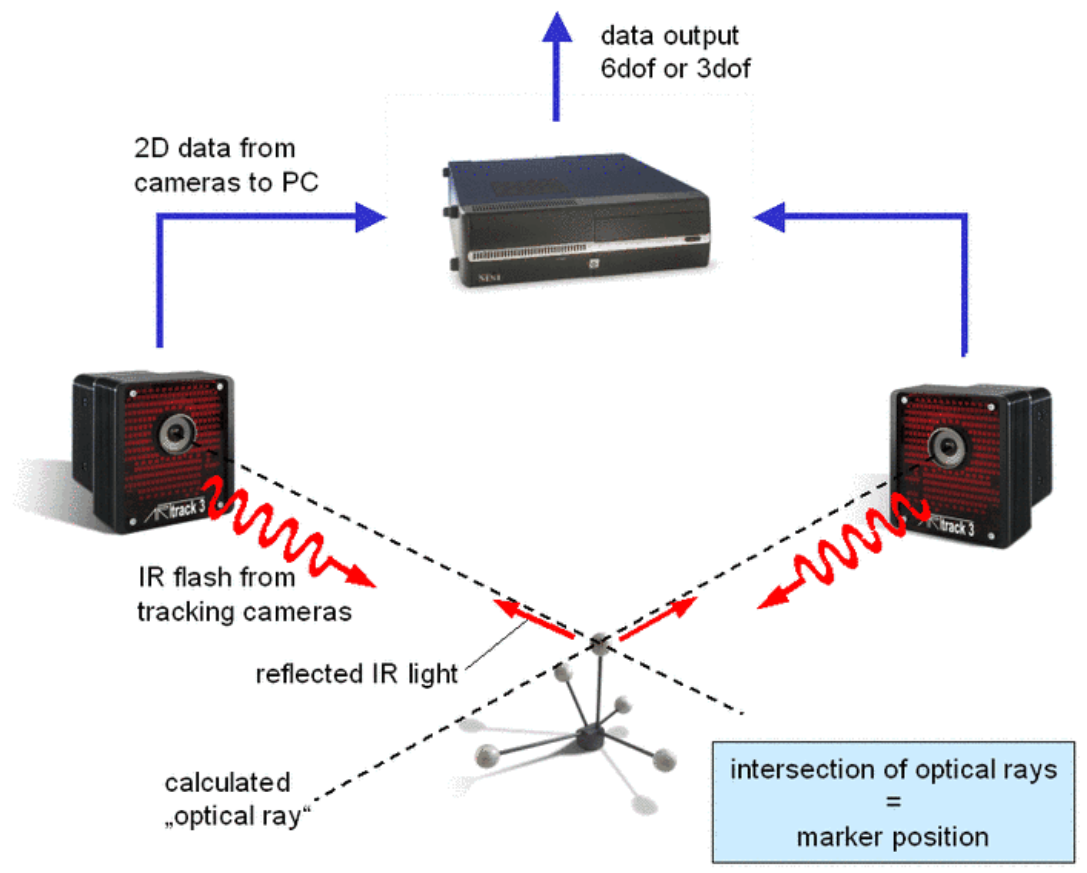

Figure 5. Functional principle of DTrack system, Image: Advanced Realtime Tracking GmbH

\section{Actuators}

The air cushion vehicles are equipped with 3 types of actuators: 12 proportional thrusters (final configuration) for position and attitude control, 3 reaction wheels for fast attitude control and an actuated linear stage to change the position of the Attitude Platform in $z$-direction.

\section{Proportional thrusters}

The Attitude Platform is equipped with 12 proportional thrusters in the final configuration and 6 proportional thrusters in the current configuration. The thrusters consist of a proportional valve and a nozzle which has been adapted for this specific application. The valves are controlled by a pulse-width modulation (PWM) signal and can be opened between 0 and $100 \%$. The PWM is generated by a microcontroller on a separate thruster control board. The thrusters are supported by 6 bar pressurized air and can generate a maximum thrust of $65 \mathrm{mN}$.

Optimization of thruster configuration The thruster configuration for the final Attitude Platform has been optimized to fulfill the following requirements:

- The Control Authority ${ }^{4,5}$ in the $x / y$-plane shall approximate a circle.

- Angular accelerations in the $z$-direction shall be the same as in the $x / y$-direction.

- Time constants for translational and rotational dynamics shall be the same. 
For easy construction it has been assumed that the thrusters are mounted in blocks of 4 at the outer ring of the Attitude Platform (see figure 6). 3 of these blocks are needed to perform full 6 degree of freedom $(6 \mathrm{DoF})$ control. Two types of configurations have been investigated. Given the mounting points of the thrusters, which depend on the design, the parameters to be optimized are the two angles describing the thrust direction:

- $\alpha$ describes the projection into the $x / y$-plane of the angle from the $x$-axis for thrusters 1-4 (configuration 3 ) or thrusters 3 and 4 (configuration 4) and similar for the other thruster blocks.

- $\beta$ describes the angle from the $x / y$-plane for thrusters 1-4 (configuration 3 ) or thrusters 1 and 2 (configuration 4) and similar for the other thruster blocks.

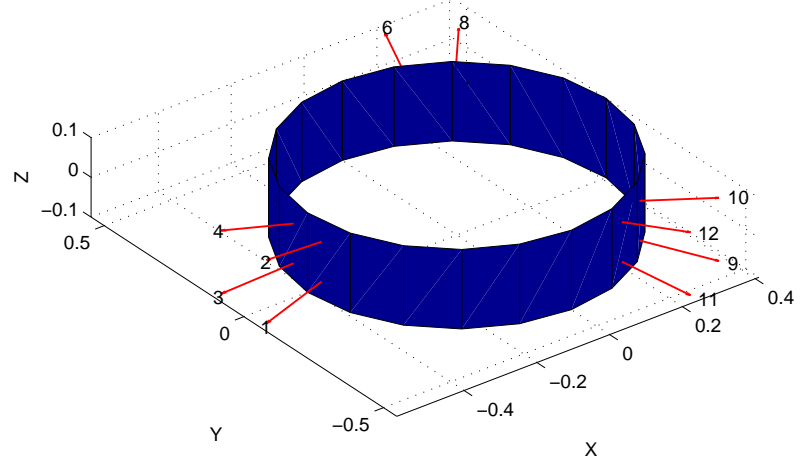

(a) Configuration 3

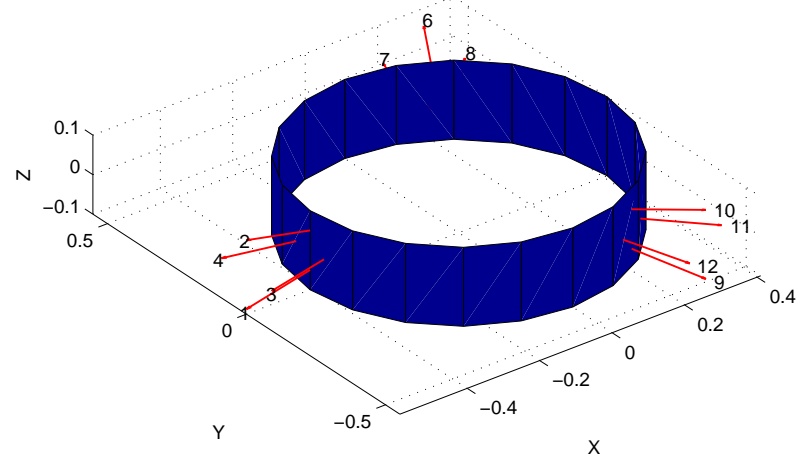

(b) Configuration 4

Figure 6. Thruster configuration

The optimization gives the result shown in table 2 and figures 7 and 8 . It can be easily seen that the differences between the two configurations are negligible. Only the force in $z$-direction shows a larger difference. As the $z$-direction points in the direction of the gravitation vector and therefore the $z$-axis can not be emulated, the force in this direction is of no importance.

Table 2. Thruster configuration optimization results

\begin{tabular}{|l|l|l|}
\hline & Configuration 3 & Configuration 4 \\
\hline$\alpha\left[^{\circ}\right]$ & 10.1 & 13.0 \\
$\beta\left[^{\circ}\right]$ & 9.4 & 11.9 \\
$F_{x, \max }[\mathrm{N}]$ & 3.89 & 3.91 \\
$F_{y, \max }[\mathrm{N}]$ & 3.71 & 3.61 \\
$F_{x, \max } / F_{z, \max }$ & 3.96 & 6.29 \\
$t[\mathrm{~s}]$ time constant & 9.40 & 9.53 \\
\hline
\end{tabular}




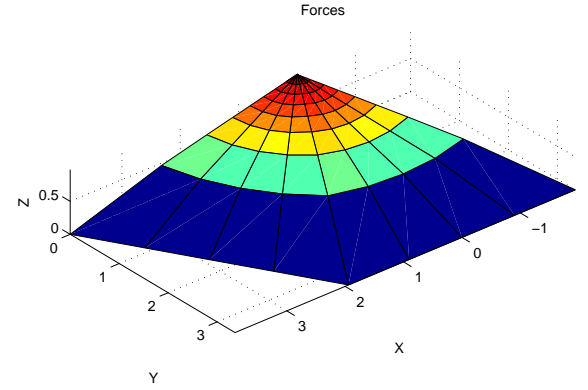

(a) Configuration 3

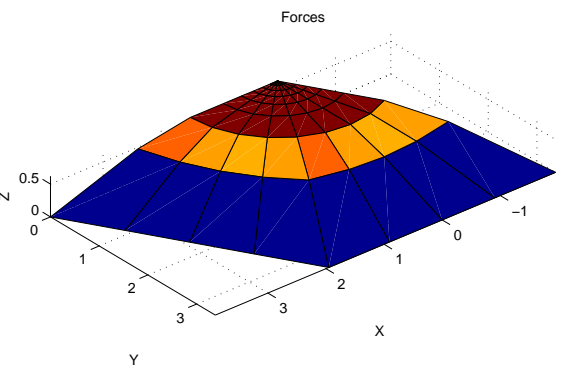

(b) Configuration 4

Figure 7. Control authorities of forces in multiples of maximum thrust per thruster

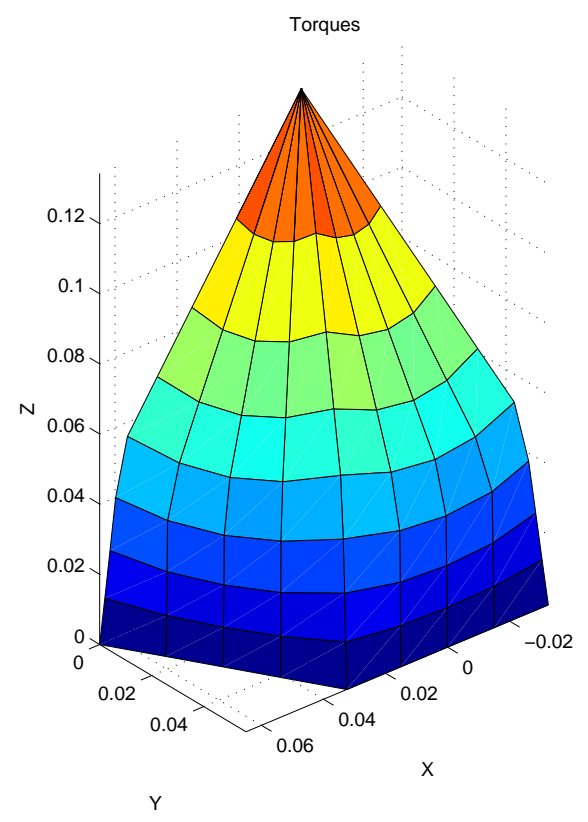

(a) Configuration 3

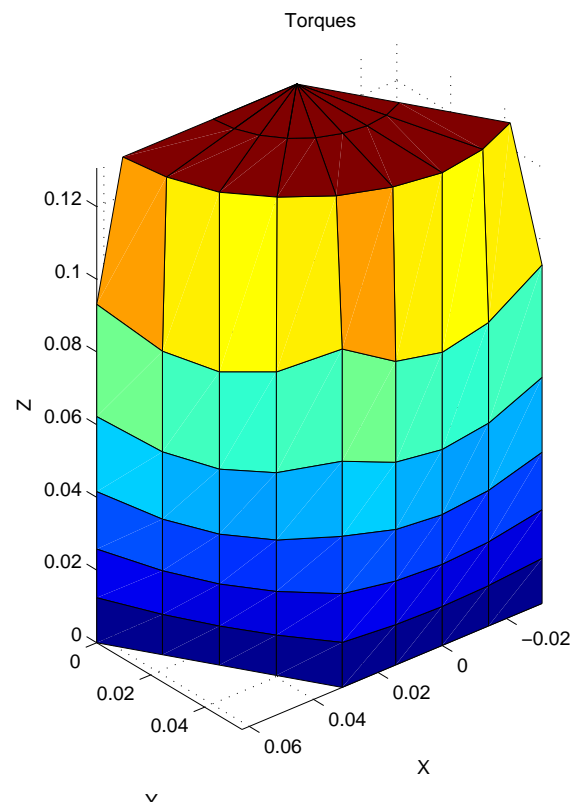

(b) Configuration 4

Figure 8. Control authorities of torques in multiples of maximum thrust per thruster per meter 


\section{Reaction wheels}

Three reaction wheels are used for fast and gas saving attitude control of the Attitude Platform. As reaction wheels the RW90 from Astro- und Feinwerktechnik Adlershof GmbH have been chosen. The technical specification of the wheels can be found in table 3 .

Table 3. RW90 technical specification

\begin{tabular}{|l|l|}
\hline Parameter & RW90 \\
\hline Angular momentum & $0.36 \mathrm{Nms} @ 7800 \mathrm{rpm}$ \\
Nominal rotation speed & $6000 \mathrm{rpm}$ \\
Max. rotation speed & ca. $7820 \mathrm{rpm}$ \\
Speed deviation $(\mathrm{rms})$ & $0.26 \mathrm{rpm}$ \\
Speed deviation $(\max )$ & $\pm 0.96 \mathrm{rpm}$ \\
Max. commandable torque & $0.015 \mathrm{Nm}$ \\
Torque deviation $(\mathrm{rms})$ & $<2 \cdot 10^{-5} \mathrm{Nm}$ \\
Torque deviation $(\max )$ & $<6 \cdot 10^{-5} \mathrm{Nm}$ \\
\hline
\end{tabular}

\section{Actuated linear stage}

Due to the gravitational acceleration the $z$-axis is not so easy to emulate. If one would like to emulate the force free motion along the $z$-axis one would need to measure the force acting on the spherical air bearing, subtract the known weight of the upper platform and the difference would be the resulting force due to the thruster or external forces. The weight of the final Attitude Platform will be about $F_{g}=m g=50 \mathrm{~kg} \cdot 9.81 \mathrm{~m} / \mathrm{s}^{2} \approx 500 \mathrm{~N}$, but the maximum thrust of one thruster is only $65 \mathrm{mN}$. This results in a ratio of about 7000 . If one would dissolve only a tenth of the maximum thruster force the ratio would be 70000 . No force sensor has such a small resolution.

So for the FFT it has been decided to artificially actuate the position along the $z$-axis. For that an actuated linear stage is mounted in the center of the Transport Platform (see figure 4(b)). With this stage it is possible to enlarge the height of the vehicles by $30 \mathrm{~cm}$. As this is not an emulation of the force free dynamics, it is not so important for the test of formation control algorithms. But to test relative navigation sensors or to perform docking maneuvers it is a necessary feature.

\section{Onboard Computer}

The onboard computer is a PC104 stack with several boards. The CPU is a x86 Atom Z530 processor with 1.6 Ghz. The CPU board also includes $1 \mathrm{~GB}$, Ethernet adapter, keyboard, mouse and VGA interface and IDE adapter. A WLAN adapter is also included as Mini PCI Express card. The WLAN connection is needed for program upload, commanding and data download. A Compact Flash card on a separate PC104 board acts as storage for operation system, application programs and data. A serial interface card is needed with 8 serial interfaces (RS232, RS422 and RS485) to connect the following components on the Attitude Platform:

- IMU

- Reaction wheels

- Thruster control board

- Actuated linear stage (via Bluetooth)

- Center of Gravity control system (see F)

- Power distribution unit (PDU). 


\section{E. Software}

The development of the application software for the air cushion vehicles is based on Matlab/Simulink. With Simulink the simulation and controller development can be done in a graphical way. Mathworks, the manufacturer of Matlab/Simulink provides also an extension called Real-Time Workshop (RTW). With the RTW it is possible to automatically generate C-code from a Simulink model. This is done for the FFT. The interface between the Simulink model and the hardware is realized by so-called S-functions. S-functions provide a mechanism to include C-code in a Simulink model. For the FFT they have been adapted manually to access the hardware driver.

As operating system for the onboard computer the real-time operating system (RTOS) QNX is used. QNX is a Unix-like, POSIX compatible RTOS for embedded systems based on a microkernel. The fact that QNX is POSIX compatible makes it easy to reuse code written for other POSIX compatible operating systems. QNX runs on many modern CPUs like PowerPC, x86 family, MIPS, SH-4 and ARM processors. The operating system comes with integrated development environment (IDE) based on the open-source IDE Eclipse and with a $\mathrm{C} / \mathrm{C}++$ compiler based on the GNU C-compiler. This makes the integration into Matlab/Simulink/RTW easy.

Matlab, Simulink and RTW as well as the QNX IDE and compiler have to be installed on one host PC. After designing the onboard algorithms with Simulink the model can be converted to C-code using RTW and compiled using the QNX C compiler. The executable is than uploaded to the onboard computer via WLAN and executed. During the execution the model running on the target processor can be connected with the original Simulink model. This makes it possible to change parameters during execution and to download, view and save real-time data from the target processor.

This process has many advantages. The path from the idea to the algorithms to a running program is very fast and automated in many parts. Simulink is the de-facto standard as tool for simulation and control design. External users can bring their own mission specific algorithms that they want to have tested and run with the Formation Flying Testbed easily without code modifications.

\section{F. Center of gravity control}

To emulate a momentum-free rotational motion with the Attitude Platform the center of gravity (CoG) of the platform must coincide with the center of the spherical air bearing. Otherwise the difference would result in a lever arm which would generate a disturbing torque due to the gravitational acceleration. The requirement to be fulfilled says that the disturbing torque shall not be larger than $T_{\text {dist }}<1 \cdot 10^{-4} \mathrm{Nm}$. Assuming a mass of the Attitude Platform of $m=50 \mathrm{~kg}$ the maximum displacement of the CoG shall not be larger than $\Delta x<2 \cdot 10^{-4} \mathrm{~mm}$.

To fulfill this requirement a 3 stage system of movable weights is foreseen:

1. Fixed weights The fixed weights will be manufactured by mass and placed at free places on the Attitude Platform. The manufacturing accuracy is assumed to be $\Delta m<50 \mathrm{~g}$ and the placing accuracy $\Delta x<50 \mathrm{~mm}$.

2. Manually adjusted weights The manually adjusted weights have a mass of $200 \mathrm{~g}$ and are mounted at the upper profiles of the Attitude Platform. They can be adjusted manually with an accuracy of $2 \mathrm{~mm}$.

3. Automatically adjusted weights These weights are adjusted be a spindle drive with an accuracy of $0.02 \mathrm{~mm}$. They have a mass of $100 \mathrm{~g}$.

A summary of masses $m$, lengths $l$ and accuracies $\Delta x$ of all 3 stages can be found in table $4 . T$ is the total torque that can be compensated with this stage, $\Delta T$ is the torque resolution. The safety factor is the factor between the torque resolution $\Delta T$ of one stage and the total torque $T$ that can be compensated by the next finer stage.

\section{G. Status}

The current status of the FFT can be seen in figures 3 and 4(a). The Transport Platform is already finished and floating above the granite table. Instead of the Attitude Platform a fixed platform is mounted. It already 
Table 4. Summary of CoG control system

\begin{tabular}{|l|l|l|l|l|l|l|}
\hline & $m[\mathrm{~g}]$ & $l[\mathrm{~mm}]$ & $\Delta x[\mathrm{~mm}]$ & $T[\mathrm{mNm}]$ & $\Delta T[\mathrm{mNm}]$ & Safety factor \\
\hline Fixed weights & 50 & & 50 & & 25 & \\
Manually adjusted weights & 200 & 200 & 2 & 400 & 4 & 16 \\
Automatically adjusted weights & 100 & 100 & $2 \cdot 10^{-2}$ & 100 & 0.02 & 25 \\
Total requirement & 50000 & $2 \cdot 10^{-4}$ & & 0.1 & & 5 \\
\hline
\end{tabular}

includes 6 thrusters for translational control in $x / y$-direction and rotational control around the $z$-axis. A DTrack target is also mounted as absolute position sensor. A fully working version of the onboard computer including operating system and application software makes it possible to control the complete vehicle in 3 dimensions. As the software tool chain (Matlab/Simulink, compiler, upload of control algorithms) is already working it is possible to design a first demonstration controller including navigation filter and thruster actuation algorithm (see also section III).

The next big step is the design of the Attitude Platform including a separate air system, the CoG control system and the spherical air bearing (see figure 4(b)). Once the Attitude Platform is working correctly we are going to build the second vehicle.

\section{H. Simulation}

A simulation of the vehicles of the Formation Flying Testbed has been set up. This simulation does not only include the dynamics of the vehicles but also disturbing forces, sensor models and actuator models. In detail the models include:

- Translational and rotational dynamics of the vehicles in current configuration and with freely rotating Attitude Platform

- Changing mass and moment of inertia

- External forces and torques

- Disturbing force due to non leveled granite table

- Disturbing torque due to offset between center of gravity and center of spherical air bearing

- Atmospheric drag

- Friction of spherical air bearing

- Sensor and actuator models

- Offset and rotation between sensor/actuator and center of gravity or body coordinate system respectively

- Mounting and alignment error

- Bias, noise, range and scale error

- Position depending systematic error of the DTrack system

- Characteristic curve of the thrusters

- Unknown systematic error of the thrusters

With this simulation it is possible to test control algorithms before applying them on the test bed. It is also interesting for external users which bring their own algorithms. With the simulation they can check principle behavior and interfaces in advance before utilizing the test bed and testing their algorithms with real dynamics. 


\section{Navigation Filter and Controller}

First algorithms for the control of the current configuration of the vehicles have been developed. They consist of three parts: navigation filter, controller and the thruster actuation algorithm. For the navigation filter and the controller the 3 degrees of freedom (translation in $x$ and $y$, rotation $\phi$ around $z$-axis) are decoupled. Therefore 3 separated filters and controllers have been designed, while the filters and the controllers for the translational degrees of freedom share the same set of parameters.

\section{A. Navigation Filter}

The navigation filter is a static gain Kalman Filter ${ }^{6}$ with the commanded forces and torques as inputs and the measured position from the DTrack as measurement. The measurements from the IMU are not used at the moment as the IMU is not part of the current configuration of the vehicles. In addition constant disturbances are estimated. For every degree of freedom a linear model is used to describe the dynamics:

$$
\underline{\dot{x}}=\underline{F} \underline{x}+\underline{B} \underline{u}+\underline{G} \underline{w}=\left[\begin{array}{ccc}
0 & 0 & 1 \\
1 & 0 & 0 \\
0 & 0 & 0
\end{array}\right] \underline{x}+\left[\begin{array}{l}
1 \\
0 \\
0
\end{array}\right] \underline{u}+\left[\begin{array}{ll}
1 & 0 \\
0 & 0 \\
0 & 1
\end{array}\right] \underline{w}
$$

with the states $\underline{x}=\left[v, x, a_{d i s t}\right]^{T}$ (velocity, position, disturbing acceleration) or $\underline{x}=\left[\omega, \phi, \alpha_{d i s t}\right]^{T}$ (angular rate, angle, disturbing angular acceleration) respectively, the input $\underline{u}=\left[a_{t h r}\right]$ (thruster acceleration) or

$\underline{u}=\left[\alpha_{t h r}\right]$ (thruster angular acceleration) and the system noise $\underline{w}$ with the system noise covariance matrix $\underline{Q}=E\left\{\underline{w} \underline{w}^{T}\right\}$. For the system noise covariance matrix a diagonal matrix is assumed:

$$
\underline{Q}=\left[\begin{array}{cc}
\sigma_{\dot{v}}^{2} & 0 \\
0 & \sigma_{\dot{a}_{d i s t}}^{2}
\end{array}\right]
$$

$\sigma_{\dot{v}}$ describes all the errors in the acceleration, mainly caused by the errors in the thruster forces. This error is set to $5 \%$ of the total thrust of one thruster. $\sigma_{\dot{a}_{\text {dist }}}$ which describes the change of the disturbing acceleration is set in such a way that the estimation of the disturbing acceleration does not affect the estimation of the position and velocity. This is the case when the time constant for the disturbance estimation is about 8 times larger than the time constant for position and velocity estimation.

The measurement equation is as follows:

$$
\underline{z}=\underline{H} \underline{x}+\underline{v}=\left[\begin{array}{lll}
0 & 1 & 0
\end{array}\right]+\underline{v}
$$

with the measurement noise $\underline{v}$ described by the measurement noise covariance matrix $\underline{R}=E\left\{\underline{v} \underline{v}^{T}\right\}=\sigma_{x}^{2}$. This noise is caused by the measurement noise of the tracking system which has a value of $\sigma_{x}=1 \mathrm{~mm}$. Designing the Kalman-Filter results in the following time constants of the closed loop navigation filter:

Table 5. Time constants and damping of navigation filter

\begin{tabular}{|l|l|l|}
\hline & Translational motion & Rotational motion \\
\hline Velocity/angular velocity & $9.9 \mathrm{~s} / 0.704$ & $12.1 \mathrm{~s} / 0.704$ \\
Position/angle & $9.9 \mathrm{~s} / 0.704$ & $12.1 \mathrm{~s} / 0.704$ \\
Disturbance acceleration/angular acceleration & $80.2 \mathrm{~s} / 1.0$ & $97.4 \mathrm{~s} / 1.0$ \\
\hline
\end{tabular}

\section{B. Controller}

The controller is a linear-quadratic regulator (LQR) controller with direct feedback of the estimated disturbance. ${ }^{7}$ For the design of the LQR controller the following linear model is used:

$$
\underline{\dot{x}}=\underline{F} \underline{x}+\underline{B} \underline{u}=\left[\begin{array}{ll}
0 & 0 \\
1 & 0
\end{array}\right] \underline{x}+\left[\begin{array}{l}
1 \\
0
\end{array}\right] \underline{u}
$$


with the states $\underline{x}=[v, x]^{T}$ (velocity, position) or $\underline{x}=[\omega, \phi]^{T}$ (angular rate, angle) respectively and the input $\underline{u}=\left[a_{t h r}\right]$ (thruster acceleration) or $\underline{u}=\left[\alpha_{t h r}\right]$ (thruster angular acceleration). With the LQR design method a static feedback gain $\underline{K}$ is computed in such a way that the cost function

$$
J=\int_{0}^{\infty} \underline{x}^{T} \underline{Q} \underline{x}+\underline{u}^{T} \underline{R} \underline{u} d t
$$

is minimized. The main criteria for the choice of the weighting matrices $Q$ and $\underline{R}$ is that the overshoot of the closed loop system should not saturate the thrusters. This results in the following time constants for the closed loop control system:

Table 6. Time constants and damping of closed loop control system

\begin{tabular}{|l|l|l|}
\hline & Translational motion & Rotational motion \\
\hline Time constant & $68.8 \mathrm{~s}$ & $44.4 \mathrm{~s}$ \\
Damping & 0.707 & 0.707 \\
\hline
\end{tabular}

\section{Thruster Actuation Algorithm}

Given the thrust vector $\tilde{T}$ describing the thrust of each thruster the forces and torques generated by the thrusters can be computed using the thruster configuration matrix $\underline{A}$ :

$$
\underline{\tilde{F}}=\underline{A} \underline{\tilde{T}}
$$

with the generalized force vector $\underline{\tilde{F}}=[\underline{F} \underline{T}]$. The problem is now to find a thrust vector that fulfills equation 6 given a commanded generalized force vector $\underline{\tilde{F}}$ coming from the controller. ${ }^{8}$

For the FFT the pseudo-inverse of the thruster configuration matrix is used:

$$
\underline{\tilde{T}}=\underline{A}^{+} \underline{\tilde{F}}=\left(\underline{A}^{T} \underline{A}\right)^{-1} \underline{A}^{T} \underline{\tilde{F}} .
$$

The pseudo-inverse also optimizes the 2-norm of the thrust vector $\underline{\tilde{T}}$.

Besides that, the thrust vector also has to fulfill the constraint that the thrust can not be negative and not larger than the maximum thrust. To modify the thrust vector computed from equation 7 such that the constraints are fulfilled, a nullspace vector can be added. A nullspace vector $\underline{N}$ is a vector that fulfills the equation

$$
\underline{A} \underline{N}=0 .
$$

By adding a nullspace vector to a thrust vector that fulfills equation 6 the new thrust vector still fulfills this equation.

For a symmetric thruster configuration like the one we have for the current and final configuration of the FFT vehicles the unit vector is always a nullspace vector. This unit vector is multiplied with the absolute value of the smallest (negative) element of $\underline{\tilde{T}}$ and added to the thrust vector. This guarantees that no element of $\underline{\tilde{T}}$ is negative.

The described method works well as long as the generalized force vector $\underline{\tilde{F}}$ is within the control authority of the thruster system. If the forces or torques are larger than the control authority they have to be limited to the control authority.

\section{Results}

\section{A. Quality of force-free environment}

To show the performance of the force and momentum-free environment a constant attitude rate is commanded to the onboard controller. The position is also controlled at the same time. As a constant set point is commanded for the position the translational velocity in steady state is zero. After reaching steady state the thrusters are turned off. The vehicle is floating force and momentum-free on the table with initial zero velocity. The results of this test are shown in figure 9 . 


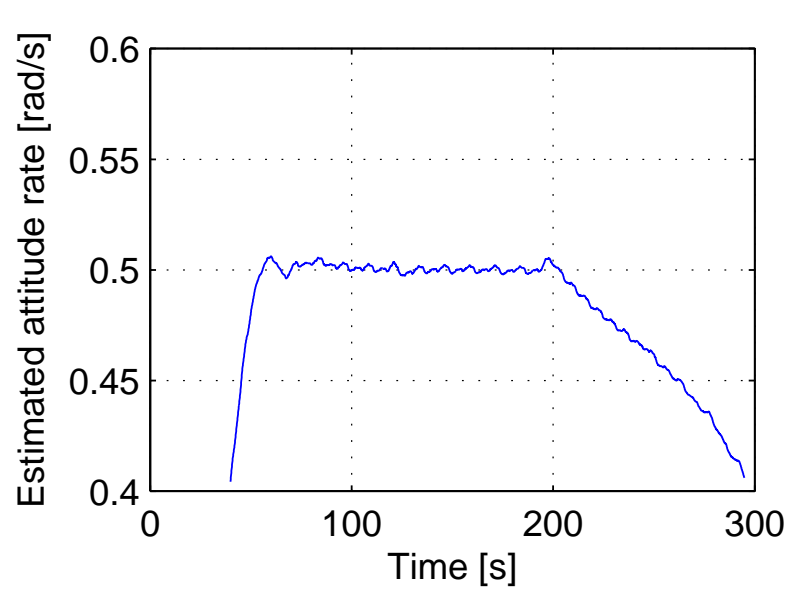

(a) Attitude rate

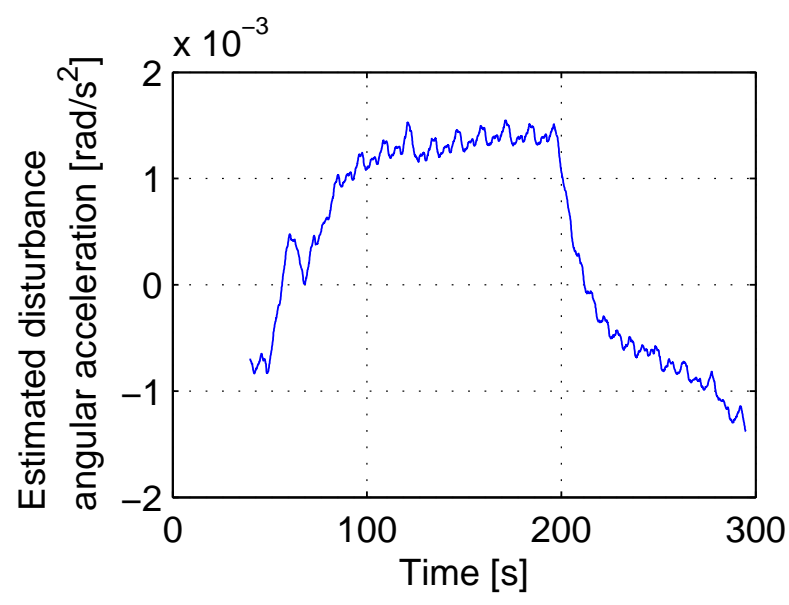

(b) Estimated disturbance angular acceleration

Figure 9. Attitude rate of free floating vehicle

As one can see a constant attitude rate of $\omega=0.5 \mathrm{rad} / \mathrm{s}$ has been commanded. The vehicle reaches steady state at $t \approx 60 \mathrm{~s}$. Using the thrusters the controller keeps the vehicle at the commanded attitude rate. The disturbance angular acceleration is estimated as $\alpha \approx 1.3 \mathrm{~m} / \mathrm{s}^{2}$. This is due to a systematic error in the thrusters characteristic.

At $t \approx 200 \mathrm{~s}$ the thrusters are turned off while the navigation filter and such the estimation of the disturbance torque is still working. One can see that the attitude rate is going down. The estimated disturbance angular acceleration $\alpha \approx-1.0 \mathrm{~m} / \mathrm{s}^{2}$ corresponds to the slope in the attitude rate plot. This is due to the friction and the drag of the free floating vehicle.

\section{B. Performance of controlled vehicles}

To show the performance of the controlled vehicles a step in the position is commanded and the step response of the plant is recorded. After reaching the final position the steady state performance of the system is also recorded. The results of the test are shown in figures 10, 11 and 12 .

Figure 10 shows the performance and the step response of the controlled vehicle. An initial set point of $[x, y, \phi]=[1.0,0.0,0.0]$ is commanded. The vehicle reaches the commanded position at $t \approx 80 \mathrm{~s}$ and the commanded attitude at $t \approx 125 \mathrm{~s}$. The long transient phase of the attitude control is mainly due to the time constant of the estimation of the disturbance angular acceleration.

At $t \approx 155 \mathrm{~s}$ a new set point of $[x, y, \phi]=[0.0,0.0,0.0]$ is commanded which is a step in the $x$-direction. The time constants to reach the new position correspond to the time constants of the linear design system in section III.B. One can also see that the control of the attitude and the $y$-axis are disturbed during the transition to the new set point. This is because of the inaccurate modeling of the thruster system, mainly due to systematic errors in the thrusters characteristics and due to mounting errors of the thrusters but also due to thruster noise. These modeling errors also show up in the residues of the navigation filter (second plot of figure 10). At the beginning and after the step in the position command, the phases when the thrusters are working heavily, the residues are much larger than in steady state. While the thruster errors dominate the size of the residues during the transient phases, the residues are dominated by the noise of the infrared tracking system in steady state. Here the size of the residues is below $0.3 \mathrm{~mm}$.

Figure 11 shows the performance of the controlled plant in steady state. One can see that the steady state control errors are below $1 \mathrm{~mm}$ in position and below $0.005 \mathrm{rad} \approx 0.3^{\circ}$ in attitude.

The transient phase of the navigation filter is shown in figure 12. Here one can see that the time constants of the residues correspond to the time constants of the linear design model in section III.A. 

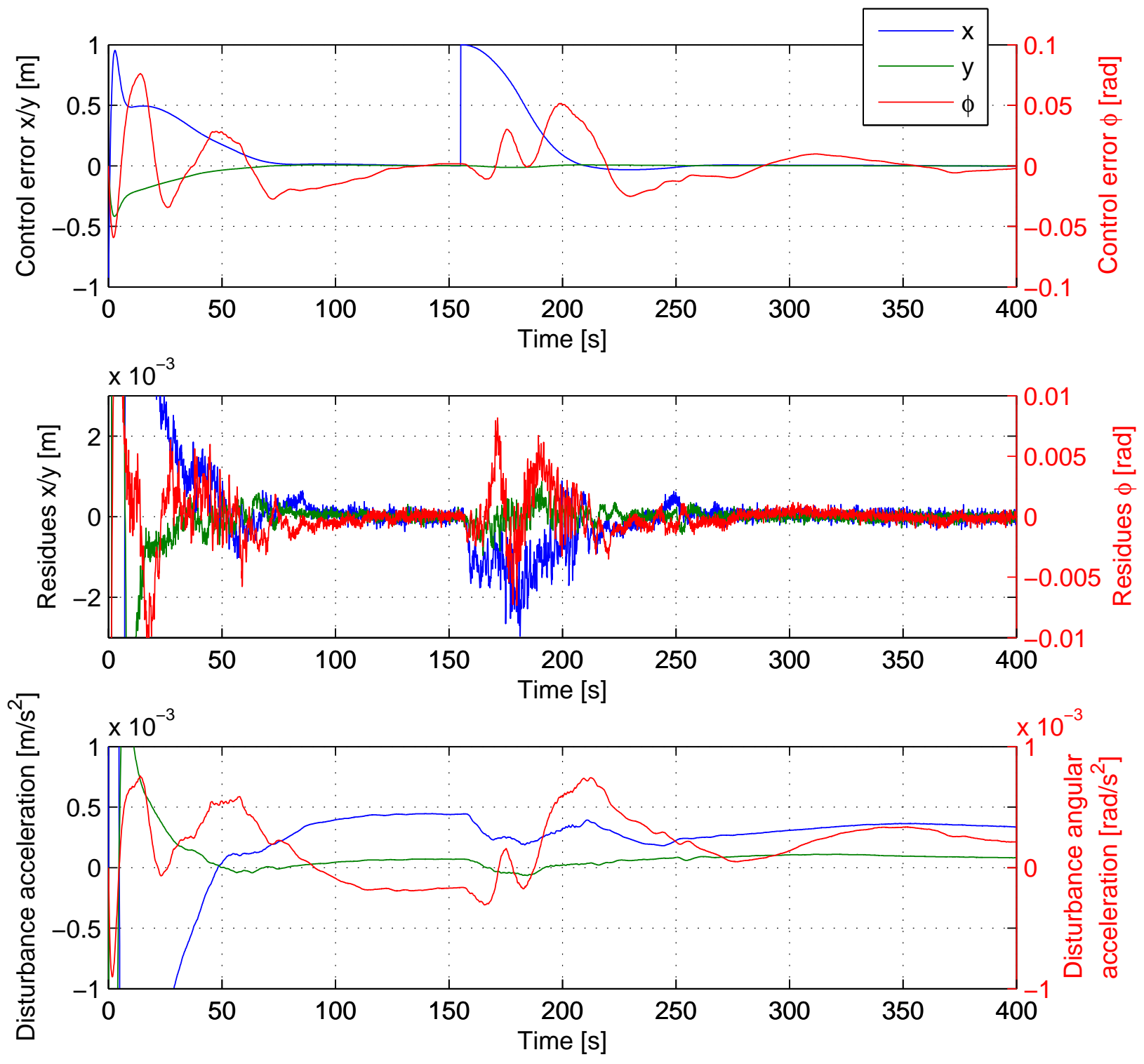

Figure 10. Performance of controlled vehicle 


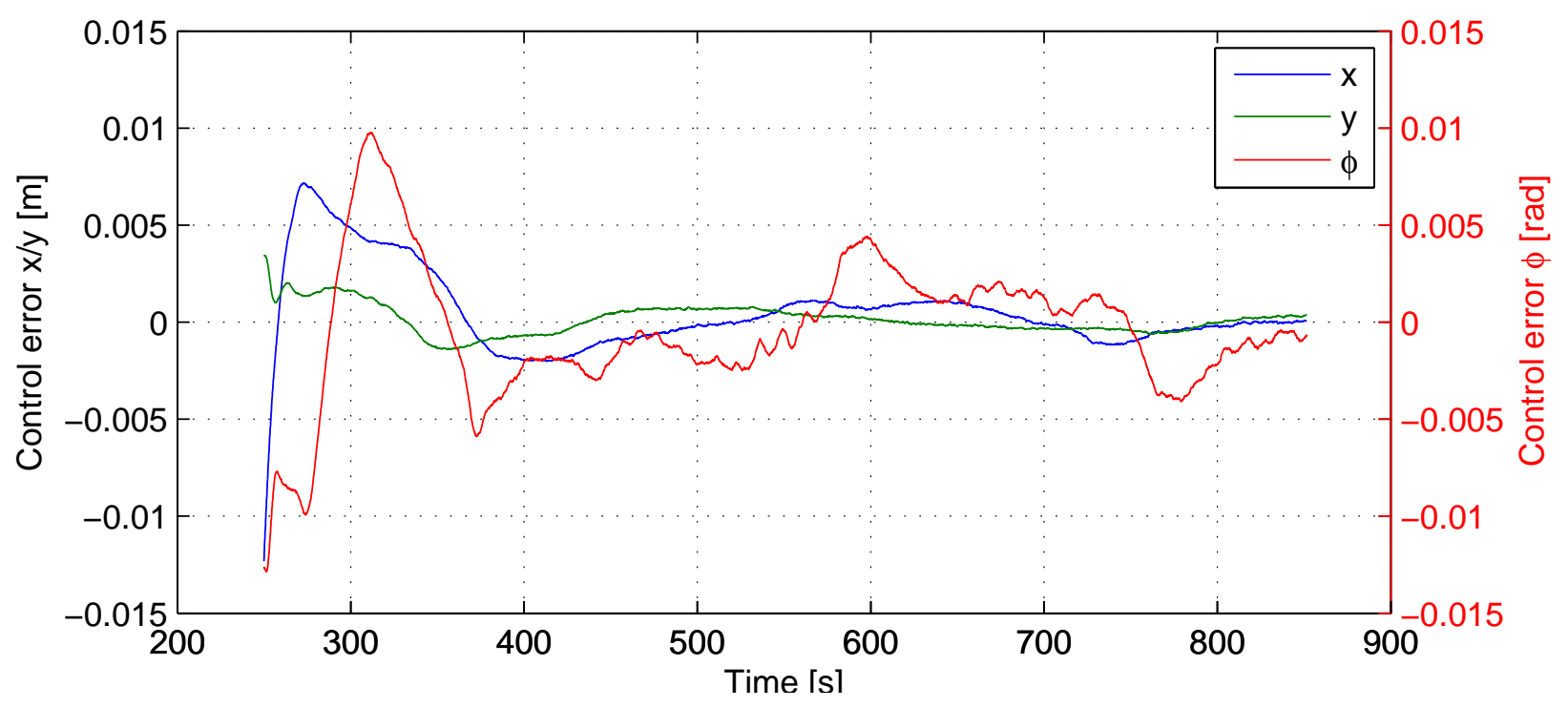

Figure 11. Steady state performance of controlled vehicle

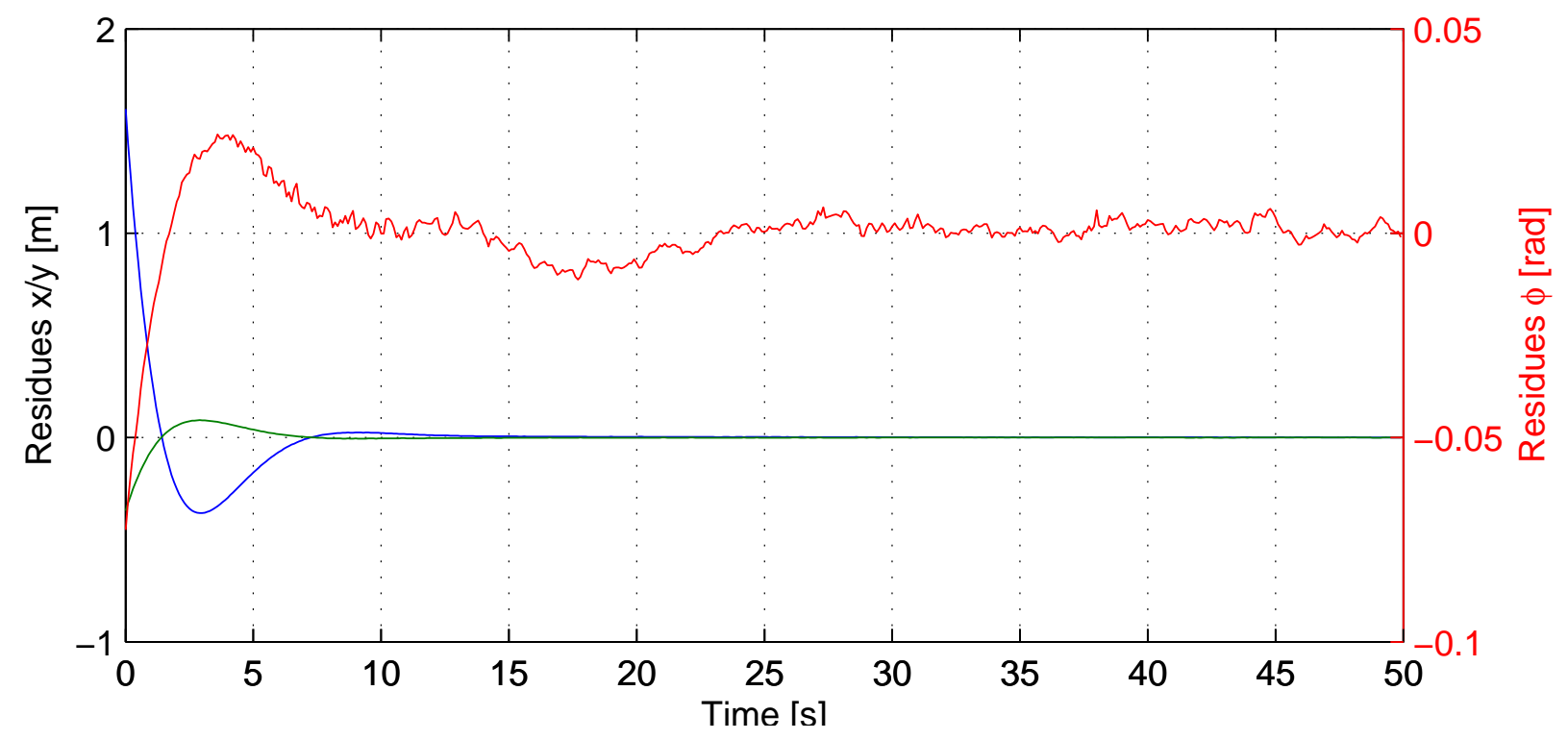

Figure 12. Transient phase of navigation filter 


\section{Conclusion and Outlook}

This paper describes the Formation Flying Testbed, a facility at the DLR Institute of Space Systems in Bremen, Germany. In the facility air cushion vehicles that float on large granite table are used to emulate the force and momentum-free environment in space. This provides the opportunity to test control algorithms and relative navigation sensors for multi-spacecraft missions, to emulate contact dynamic behavior for missions that use docking or grabbing and to emulate the dynamics of spacecrafts that use manipulator arms.

The assembly of the facility is described with a focus on the design of the air cushion vehicles. The sensors and actuators used to control the vehicles are shown as well as other components to make the vehicles work like the air system and the onboard computer. The software toolchain used to design and implement the onboard algorithms is illustrated along with the simulation used to test control algorithms before implementing them on the vehicles.

A focus is laid on the design of the onboard algorithms including navigation filter, controller and thruster actuation algorithm. Measurements from the controlled vehicle show the quality of the force and momentumfree environment and the performance of the controller and navigation filter.

At the moment a prototype vehicle is finished with a fixed upper platform. Within this year the rotating Attitude Platform will be assembled to emulate also the 3 rotational degrees of freedom. If this works well, everything is prepared to build a second vehicle with the same configuration as the first one.

In addition the facility will be expanded by four smaller air cushion vehicles. These vehicles will be capable to float on the table and to rotate around the $z$-axis but not to rotate in all three rotational degrees of freedom. In difference to the larger vehicles they will not have an IMU and reaction wheels. These smaller vehicles will be used for simulation of swarms and distributed intelligence. They are manufactured by the Center of Applied Space Technology and Microgravity (ZARM) at the University of Bremen, Germany.

At the beginning of the next year the facility will move to a new building. There it will be extended by a second granite table to enlarge the experiment space to a total size of $5 \mathrm{~m} \times 4 \mathrm{~m}$.

\section{References}

\footnotetext{
${ }^{1}$ DARWIN homepage, October 2009, http://www.esa.int/science/darwin.

${ }^{2}$ XEUS homepage, August 2009, http://www.esa.int/science/xeus.

${ }^{3}$ DEOS homepage, http://www.spacetech-i.com/deutsche-orbitale-servicing-mission.html; visited on June 2010.

${ }^{4}$ Wiktor, P. J., "Minimum Control Authority Plot: A Tool for Designing Thruster Systems," AIAA Journal of Guidance, Control and Dynamics, Vol. 17, No. 5, September 1994, pp. 998 - 1006.

${ }^{5}$ Bindel, D., "Thruster Actuation Algorithms for Scientific Space Missions," 56th International Astronautical Congress of the International Astronautical Federation, IAF, September 2005, IAC-05-C1.P.10.

${ }^{6}$ Grewal, M. S. and Andrews, A. P., Kalman Filtering - Theory and Practice using MATLAB, John Wiley \& Sons, Second

${ }^{7}$ Föllinger, O., Regelungstechnik, Hüthig Buch Verlag Heidelberg, 8th ed., 1994.

${ }^{8}$ Wiktor, P. J., The Design of a Propulsion System Using Vent Gas from a Liquid Helium Cryogenic System, Ph.D. thesis, Department of Aeronautics and Astronautics of Stanford University, June 1992.

${ }^{9}$ Regher, M. W., Acikmese, A. B., Ahmed, A., Aung, M., Bailey, R., Bushnell, C., Clark, K. C., Hicke, A., Lytle, B., MacNeal, P., Rasmussen, R. E., Shields, J., and Singh, G., "The Formation Control Testbed," IEEE Aerospace Conference Proceedings, 2004.
} ed., 2001. 